\title{
Avaliação da Sensibilidade de Diversas Espécies de Plantas Daninhas Aquáticas ao Carfentrazone-Ethyl, em Ambiente CONTROLADO ${ }^{1}$
}

\author{
Assessment of Sensitivity of Several Aquatic Weeds to Carfentrazone-Ethyl under Controlled \\ Environment
}

FOLONI, L.L. ${ }^{2}$ e PITELLI, R.A. ${ }^{3}$

\begin{abstract}
RESUMO - Plantas aquáticas, especialmente macrófitas, tornam-se sério problema em hidrelétricas, afetando a múltipla utilização dos corpos d'água, incluindo produção de peixes e atividades de pesca, perdas d'água por evapotranspiração, esportes aquáticos, canoagem, irrigação e produção de energia nas usinas hidrelétricas. Com o objetivo de analisar o potencial de uso do carfentrazone-ethyl no controle das principais plantas daninhas aquáticas no Brasil, foi instalado um experimento em vasos com água. Utilizaram-se os seguintes tratamentos herbicidas (g i.a. ha ${ }^{-1}$ ): carfentrazone-ethyl a 15, 30 e 60; glyphosate a 4.536; 2,4-D a 4.690; imazapyr a 1.250; e uma testemunha sem herbicida. Esses tratamentos foram testados nas seguintes espécies: Eichhornia crassipes, Salvinia auriculata, Pistia stratiotes, Myriophyllum aquaticum, Brachiaria arrecta, Hydrocotyle umbellata, Typha sp. e Echinochloa polystachya. As avaliações foram efetuadas aos 7, 14, 21 e 28 dias após os tratamentos. Os resultados mostraram que o carfentrazone-ethyl foi eficiente no controle de E. crassipes (maior dose) e $P$. stratiotes (duas maiores doses), com efeito supressivo sobre S. auriculata. Foi observado que nas outras plantas daninhas estudadas não houve eficiência de controle.
\end{abstract}

Palavras-chave: herbicida, controle de plantas aquáticas, macrófitas.

\begin{abstract}
Aquatic weeds, especially macrophytes, are a serious problem in hydroelectric plant systems, affecting the multiple use of water, such as fish production and fishing activities, water losses by evapotranspiration, aquatic sports, boating, irrigation and hydroelectric power production. An experiment using boxes filled with water was carried out to analyze the potential use of carfentrazone-ethyl in the control of the main aquatic weeds in Brazil. The following herbicide treatments (g i.a. ha $\mathrm{h}^{-1}$ ) were used: carfentrazone-ethyl at 15, 30 and 60; glyphosate at 4,536; 2,4-D at 4,690; imazapyr at 1,250, and no herbicide. These treatments were tested on the species: Eichhornia crassipes, Salvinia auriculata, Pistia stratiotes, Myriophyllum aquaticum, Brachiaria arrecta, Hydrocotyle umbellata, Typha sp. and Echinochloa polystachya. The evaluations were conducted at 7, 14, 21 and 28 days after treatments. The results showed that carfentrazone-ethyl is efficient in the control of $\boldsymbol{E}$. crassipes (higher rate), $\boldsymbol{P}$. stratiotes (the two highest rates) having a suppressive effect on S. auriculata. No efficacy was observed on the other weeds studied.
\end{abstract}

Key words: herbicide, aquatic weed control, macrophytes.

Recebido para publicação em 14.1.2005 e na forma revisada em 17.3.2005.

2 Professor Dr. Colab., Dep. de Engenharia Agrícola, FEAGRI/ UNICAMP, Caixa Postal 6011, 13083-970 Campinas-SP, $<$ lfoloni@aol.com>. ${ }^{3}$ Professor Titular, Dep. de Biologia Aplicada à Agropecuária, FCAV/UNESP, Rod. Prof. Paulo D. Castellane s/n, 14807-000 Jaboticabal, <pitelli@fcav.unesp.br>. 


\section{INTRODUÇÃO}

De forma geral, quando um ecossistema não sofre perturbação, os indivíduos que o compõem estão em equilíbrio, não havendo dominância de uma determinada espécie. Em razão das alterações, dependendo da sua magnitude, pode ocorrer a predominância de determinada espécie, acontecendo, em função disso, vários problemas. Nos sistemas aquáticos, em razão da poluição, das alterações dos níveis dos lagos e represas, ou por outras atividades promovidas pelo homem, pode ser observado o efeito do desequilíbrio, externado pela presença de plantas daninhas aquáticas que até então passavam despercebidas ou não se mostravam problemáticas.

Tanaka (2001) afirma que os ambientes aquáticos, de forma geral, são formados por uma grande biodiversidade vegetal, que, em situação ecologicamente equilibrada, é essencial para a manutenção e o desenvolvimento desse ecossistema. Nesses ambientes, as plantas aquáticas são responsáveis pela oxigenação e depuração da água, servem de alimento para peixes e aves e protegem as margens da ação erosiva da água.

Segundo Pitelli (1998), no Brasil, as plantas aquáticas representam grandes problemas em três ambientes alterados pelo homem: lagos e reservatórios eutrofizados próximos a centros urbanos; represas rurais e canais de irrigação e de drenagem; e grandes reservatórios de usinas hidrelétricas. Dentro da mesma linha, Patton \& Starnes (1970) declararam que o excesso de nutrientes - provenientes de fontes como o esgoto doméstico, a erosão de terras agrícolas, os resíduos industriais e a decomposição de plantas e de outros organismos - possibilita que ambientes hídricos se tornem infestados por plantas aquáticas.

Uma grande diversidade de plantas aquáticas pode ser encontrada vegetando as margens de rios e reservatórios ou dentro dos mais diversos ambientes aquáticos, empregando diferentes mecanismos de adaptação para sobrevivência e desenvolvimento. Enquanto algumas espécies se apresentam enraizadas em corpos-d'água com fortes correntezas, outras somente podem viver em águas paradas ou estagnadas (Martins et al., 2002).

Planta Daninha, Viçosa-MG, v. 23, n. 2, p. 329-334, 2005
Cardoso et al. (2003) ressaltam que as macrófitas aquáticas em desequilíbrio nos corpos hídricos apresentam taxa de crescimento elevada e produzem grandes quantidades de biomassa. De acordo com Branco (1986), existem várias espécies macrófitas com potencial para causar prejuizos em ecossistemas aquáticos. Carvalho et al. (2003) identificaram as plantas aquáticas e os níveis de infestação de cada espécie presente no reservatório de Barra Bonita-SP, encontrando em porcentagens superiores a $10 \%$ as seguintes espécies: Brachiaria mutica, Brachiaria subquadripara e Eichhornia crassipes; e entre 7 e 9,9\%: Enidra sexxilis, Pistia stratiotes e Polygonum lapathifolium; além de outras 11 espécies encontradas em menor porcentagem.

Proporcionalmente aos recursos hídricos do Brasil, são poucos os ambientes eutrofizados que apresentam sérios problemas com plantas aquáticas. Os exemplos mais flagrantes localizam-se nas proximidades de grandes centros urbanos, como São Paulo, Belo Horizonte, Rio de Janeiro e Recife. Nesses ambientes predominam Eichhornia crassipes, Pistia stratiotes, Salvinia spp., entre outras. Essas plantas causam sérios problemas de procriação de mosquitos e de vetores de doenças humanas, impedem atividades de recreação e de pesca e proporcionam condições microaerofilicas, com produção de substâncias de ruim odor (Pitelli, 1998).

Guimarães et al. (2003) ressaltam que é cada vez maior a utilização de produtos químicos em ambientes aquáticos, principalmente em situações nas quais macrófitas aquáticas estão presentes; essa presença tem causado diversos problemas, como obstrução de turbinas e grades de proteção, funcionamento anormal de eclusas, danos ao ambiente, entraves à navegação, abrigo de vetores de várias doenças, além de prejudicar atividades de lazer.

A utilização de controle químico das plantas daninhas aquáticas no Brasil foi iniciada no final dos anos $60 \mathrm{com}$ o 2,4-D e o diquat. Martins (1998) destaca que, em termos mundiais, esse é o método de controle mais utilizado, através de aplicação de produtos como o 2,4-D, glyphosate, diquat e endothal, compostos à base de cobre, fluridone e imazapyr. 
Segundo Cardoso et al. (2003), o controle químico promove resultado rápido, sendo uma alternativa de manejo que mantém as plantas daninhas aquáticas abaixo do nível de dano econômico, não prejudicando as funções dos corpos hídricos. Outra vantagem proporcionada por controle é a especificidade, que elimina o risco de coleta indevida de animais, como ocorre na retirada de plantas dos corpos hídricos utilizando-se o controle mecânico.

Dos mais de 200 diferentes herbicidas registrados (diferentes principios ativos) nos Estados Unidos, seis deles são registrados para uso aquático. Os herbicidas 2,4-D, diquat, complexos de cobre e endothal têm sido utilizados desde 1940-50; fluridone e glyphosate foram desenvolvidos em 1970; e triclopyr e imazapyr (usados em florestas e auto-estradas) estão sendo avaliados para uso aquático (Haller, 1998). Ainda segundo Cardoso et al. (2003), por melhor conciliar eficiência e custo, o método de manejo para controle de plantas daninhas aquáticas mais difundido é a utilização dos herbicidas 2,4-D, glyphosate e diquat.

Nos anos recentes, foi introduzido no mercado brasileiro o carfentrazone-ethyl, herbicida com excelente perfil ecotoxicológico; dessa forma, o objetivo do presente trabalho foi o de avaliar a sensibilidade de diversas espécies aquáticas ao carfentrazone-ethyl, uma espécie de screening, para observar seu futuro potencial, em ambiente controlado.

\section{MATERIAL E MÉTODOS}

O presente ensaio foi conduzido na área experimental do Laboratório Giorgio De Marinis, do Departamento de Biologia Aplicada à Agropecuária da Faculdade de Ciências
Agrárias e Veterinárias, UNESP, campus de Jaboticabal, em área controlada. Foram realizadas duas baterias de teste. Na primeira, as espécies de macrófitas utilizadas foram: Eichhornia crassipes, Salvinia auriculata, Pistia stratiotes, Myriophyllum aquaticum e Brachiaria arrecta. Na segunda, usaram-se as espécies Hydrocotyle umbelatta, Typha angustifolia e Echinochloa polystachya.

Estas espécies foram plantadas em vasos com capacidade para 5 litros, os quais foram preenchidos com substrato contendo areia, turfa e água $(1,5: 1,5: 1)$. A adubação nos vasos foi feita com a fórmula comercial 04-14-08. O teor de água presente nos vasos foi reposto a cada dois dias ou toda vez em que se notava o abaixamento no nivel normal da água. Os tratamentos experimentais estudados constaram da aplicação dos herbicidas descritos na Tabela 1.

Os tratamentos herbicidas foram pulverizados respectivamente em 26/06/2002 e 23/07/2002, em área total da parcela, empregando equipamento de precisão a gás carbônico $\left(\mathrm{CO}_{2}\right)$, da marca comercial R\&D Sprayers, provido de barra compensada contendo quatro bicos de jato plano marca TeeJet XR 110.02, espaçados entre si de $0,50 \mathrm{~m}$, promovendo 2,0 $\mathrm{m}$ de largura efetiva. $\mathrm{O}$ equipamento foi operado a $2,78 \mathrm{kgf} \mathrm{cm}^{-2}$ (10 psi), empregando água como diluente e volume de aplicação de $200 \mathrm{~L} \mathrm{ha}^{-1}$. Tais bicos, segundo seu fabricante, Spraying Systems CO. (USA), operando nessas condições, produzem uma pulverização com gotas de diâmetro mediano volumétrico (DMV) de $222 \mu \mathrm{m}$ e um espectro de gotas com diâmetros variando de 74 a $550 \mu \mathrm{m}$, nos volumes acumulativos de 2 a $98 \%$, respectivamente (Spraying Systems CO., 1992).

Tabela 1 - Descrição dos tratamentos experimentais utilizados nas duas baterias de testes. FCAJV-UNESP, Jaboticabal, 2002

\begin{tabular}{|c|c|c|c|c|c|}
\hline \multirow{2}{*}{ Tratamento } & \multirow{2}{*}{ Ingrediente ativo } & \multirow{2}{*}{ Produto comercial } & \multicolumn{2}{|c|}{ Dose } & \multirow{2}{*}{ Modo de aplicação } \\
\cline { 3 - 5 } & & & g i.a. ha ${ }^{-1}$ & L p.c. ha $^{-1}$ & \\
\hline 1 & carfentrazone-ethyl & Aurora 400 CE & 15 & 0,037 & Pós \\
\hline 2 & carfentrazone-ethyl & Aurora 400 CE & 30 & 0,075 & Pós \\
\hline 3 & carfentrazone-ethyl & Aurora 400 CE & 60 & 0,150 & Pós \\
\hline 4 & glyphosate & Rodeo & 4.536 & 7,0 & Pós \\
\hline 5 & 2,4-D & DMA 806 BR & 4.690 & 7,0 & Pós \\
\hline 6 & imazapyr & Arsenal 250 & 1.250 & 5,0 & Pós \\
\hline 7 & Testemunha & & - \\
\hline
\end{tabular}


As condições climáticas no momento da aplicação foram: umidade relativa (UR\%) de $68 \%$ e $51 \%$, nebulosidade zero, vento calmo a ausente (zero a $2 \mathrm{~km} \mathrm{~h}^{-1}$ ), temperatura do ar de 22 e $26^{\circ} \mathrm{C}$ e momento da aplicação de $17 \mathrm{~h} 50$ às $18 \mathrm{~h} 20$ e $16 \mathrm{~h} 30$ às $16 \mathrm{~h} 45$, respectivamente para a primeira e segunda baterias de teste.

O delineamento experimental adotado foi o inteiramente casualizado, com sete tratamentos e quatro repetições. A suscetibilidade das plantas aos herbicidas foi avaliada de forma visual. Adotou-se a escala ALAM (1974), a qual varia entre 1 e 6 , sendo, respectivamente, as denominações de controle de: nenhum/pobre, regular, suficiente, bom, muito bom e excelente. As avaliações foram realizadas aos 7, 14, 21 e 28 dias após a aplicação (DAA) dos diferentes herbicidas. Os resultados obtidos foram submetidos à análise da variância pelo teste $\mathrm{F}$, e as médias dos tratamentos foram comparadas pelo teste t a $5 \%$ de probabilidade.

\section{RESULTADOS E DISCUSSÃO}

Os dados sumarizados nas Figuras de 1 a 8 resumem as médias das quatro repetições relativas aos valores de sensibilidade sobre as plantas daninhas, nas avaliações efetuadas aos 7, 14, 21 e 28 DAA. Para a planta Eichhornia crassipes, a dose de 15 g i.a. ha ${ }^{-1}$ do carfentrazone-ethyl foi pobre; contudo, na dose de $30 \mathrm{~g}$ i.a. ha $\mathrm{h}^{-1}$ foi observado um bom controle e, para a dose de $60 \mathrm{~g}$ i.a. ha ${ }^{-1}$, um excelente nivel de controle aos 28 DAA. Para glyphosate, 2,4-D e imazapyr foram observados excelentes controles; para este último ocorreram novas brotações aos 28 DAA (Figura 1).

No caso da espécie Salvinia auriculata, cujos dados estão apresentados na Figura 2, detectou-se pequena sensibilidade à menor dose do carfentrazone-ethyl, atribuindo-se, ao final do experimento nenhum controle. Nas doses de 30 e 60 g i.a. ha ${ }^{-1}$ foi observado bom e muito bom controle, porém com novas brotações aos 21 DAA. Os herbicidas glyphosate e imazapyr proporcionaram excelente sensibilidade, enquanto o 2,4-D foi regular, apresentando novas brotações aos 28 DAA.

A planta daninha Pistia stratiotes mostrou baixa sensibilidade ao carfentrazone-ethyl na menor dose testada, pois foram observadas novas brotações. Nas doses de 30 e 60 g i.a. ha ${ }^{-1}$ a sensibilidade foi excelente já aos 7 DAA. Para glyphosate e imazapyr foi observado excelente controle, enquanto para o 2,4-D não houve sensibilidade (Figura 3). Myriophyllum aquaticum não mostrou sensibilidade, ou esta foi pobre, para as diferentes doses avaliadas do carfentrazone-ethyl, ao passo que esta planta demonstrou alta suscetibilidade aos demais produtos utilizados (Figura 4).

Na Figura 5 são mostrados os resultados de sensibilidade para a planta Brachiaria arrecta. De forma geral, o carfentrazone-ethyl tem eficiência sobre algumas plantas daninhas de folhas largas e nenhuma sobre folhas

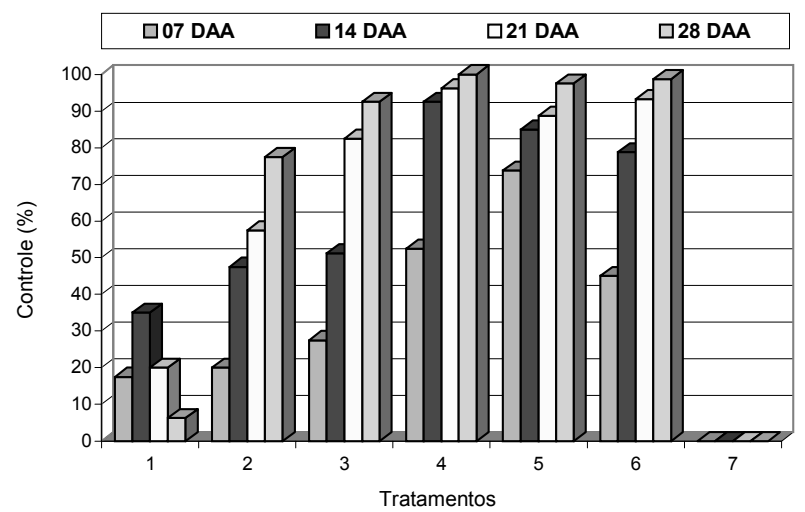

Figura 1 - Porcentagem de controle (\%) de Eichhornia crassipes (ALAM, 1974) aos 7, 14, 21 e 28 dias após aplicação (DAA) de herbicidas em pós-emergência no controle de plantas daninhas aquáticas. NEPEAM-FCAJV/UNESP, Jaboticabal, 2002.

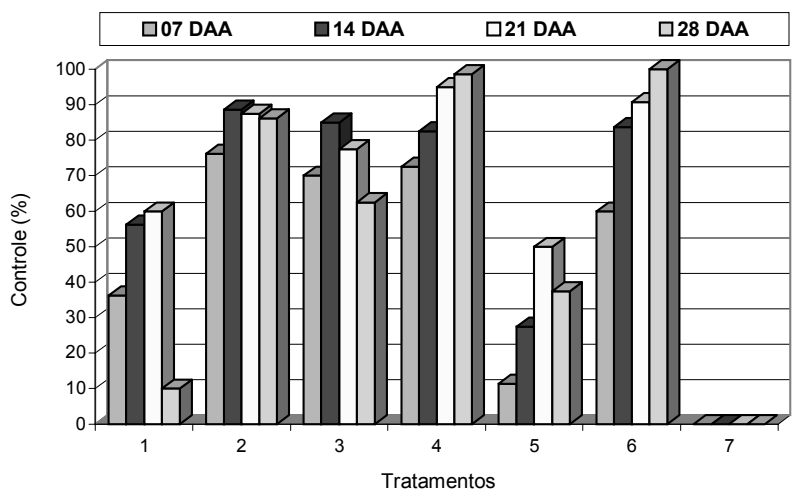

Figura 2 - Porcentagem de controle (\%) de Salvinia auriculata (ALAM, 1974) aos 7, 14, 21 e 28 dias após aplicação (DAA) de herbicidas em pós-emergência no controle de plantas daninhas aquáticas. NEPEAM-FCAJV/UNESP, Jaboticabal, 2002. 
estreitas. Os resultados sobre $B$. arrecta mostraram essa tendência: nenhuma sensibilidade foi observada para o carfentrazone. Da mesma forma, esses resultados mantiveramse para o herbicida 2,4-D, enquanto a espécie foi altamente suscetivel ao glyphosate e ao imazapyr.

Conforme pode ser observado na Figura 6, os dados de sensibilidade da planta daninha Hydrocotyle umbelatta foram nenhum ou pobre para o carfentrazone-ethyl, mostrando uma boa sensibilidade num primeiro momento para a maior dose, mas retornando posteriormente ao patamar de mal controle; já para os demais tratamentos esta planta demonstrou alta suscetibilidade. No caso da planta daninha

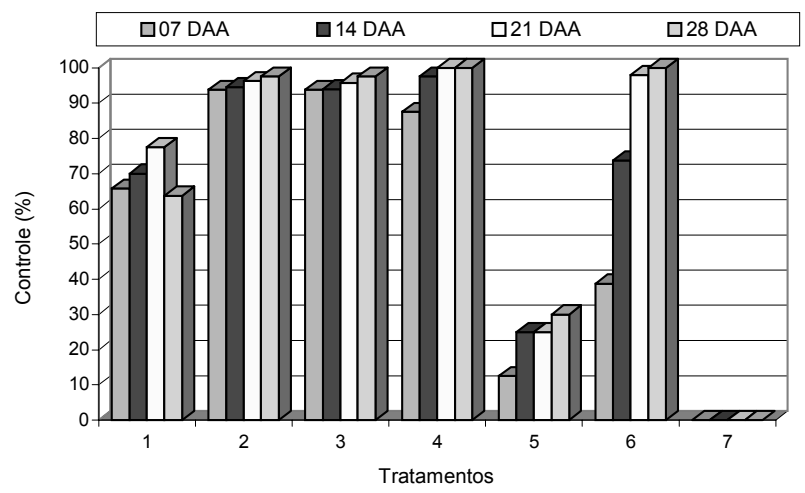

Figura 3 - Porcentagem de controle (\%) de Pistia stratiotes (ALAM, 1974) aos 7, 14, 21 e 28 dias após aplicação (DAA) de herbicidas em pós-emergência no controle de plantas daninhas aquáticas. NEPEAM-FCAJV/UNESP, Jaboticabal, 2002.

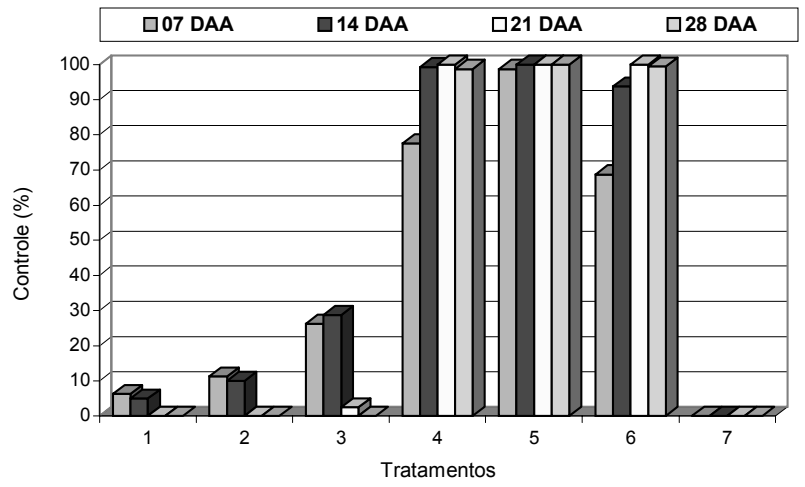

Figura 4 - Porcentagem de controle (\%) de Myriophyllum aquaticum (ALAM, 1974) aos 7, 14, 21 e 28 dias após aplicação (DAA) de herbicidas em pós-emergência no controle de plantas daninhas aquáticas. NEPEAM-FCAJV/ UNESP, Jaboticabal, 2002.
Typha sp., os resultados observados novamente demonstram baixa sensibilidade (nenhum ou pobre) para o carfentrazone-ethyl, enquanto apresentou alta sensibilidade aos demais tratamentos testados (Figura 7). Para a última planta daninha avaliada, os resultados obtidos mostraram que a sensibilidade de Echinochloa polystachya ao carfentrazone-ethyl foi pobre, regular para o 2,4-D, bom para o imazapyr e total para o glyphosate (Figura 8).

Com base nos resultados obtidos no presente experimento, pode-se considerar que o carfentrazone-ethyl tem potencial de uso no controle de macrófitas aquáticas, pois identificou-se que as espécies Eichhornia crassipes, Pistia stratiotes e Salvinia auriculata

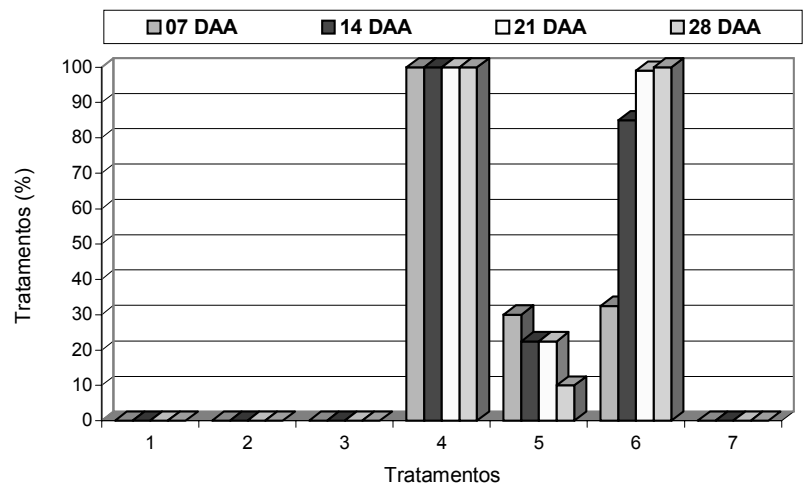

Figura 5 - Porcentagem de controle (\%) de Brachiaria arrecta (ALAM, 1974) aos 7, 14, 21 e 28 dias após aplicação (DAA) de herbicidas em pós-emergência no controle de plantas daninhas aquáticas. NEPEAM-FCAJV/UNESP, Jaboticabal, 2002.

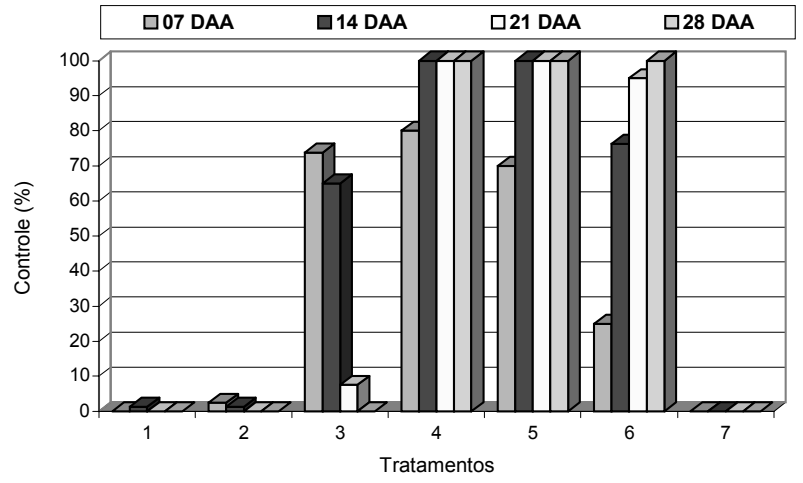

Figura 6 - Porcentagem de controle (\%) de Hydrocotyle umbelatta (ALAM, 1974) aos 7, 14, 21 e 28 dias após aplicação (DAA) de herbicidas em pós-emergência no controle de plantas daninhas aquáticas. NEPEAM-FCAJV/ UNESP, Jaboticabal, 2002.

Planta Daninha, Viçosa-MG, v. 23, n. 2, p. 329-334, 2005 


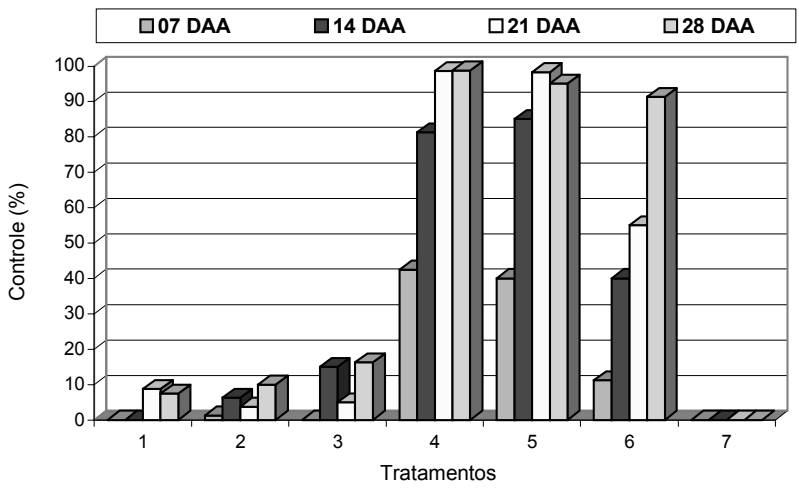

Figura 7 - Porcentagem de controle (\%) de Typha sp. (ALAM, 1974) aos 7, 14, 21 e 28 dias após aplicação (DAA) de herbicidas em pós-emergência no controle de plantas daninhas aquáticas. NEPEAM-FCAJV/UNESP, Jaboticabal, 2002.

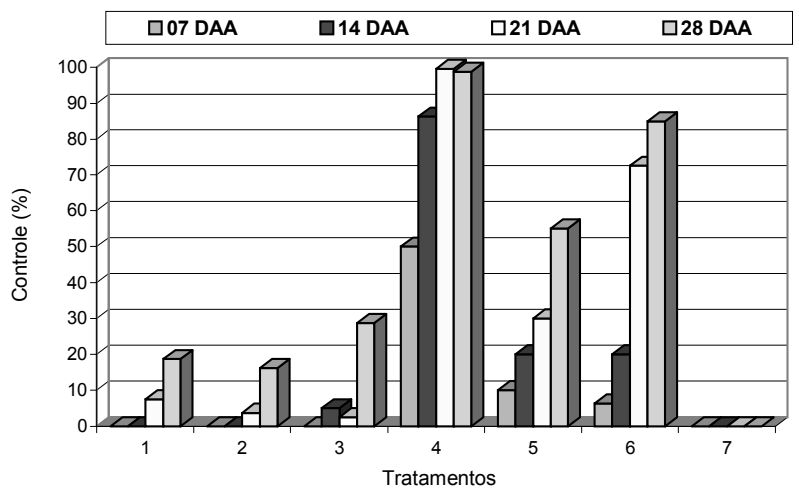

Figura 8 - Porcentagem de controle (\%) de Echinochloa polystachya (ALAM, 1974) aos 7, 14, 21 e 28 dias após aplicação (DAA) de herbicidas em pós-emergência no controle de plantas daninhas aquáticas. NEPEAM-FCAJV/ UNESP, Jaboticabal, 2002.

apresentam sensibilidade a algumas doses utilizadas. Já as espécies $M$. aquaticum, $B$. arrecta, $H$. umbellata, Typha sp. e E. polystachya não mostraram sensibilidade a este herbicida. Em razão do perfil ecotoxicológico favorável do carfentrazone-ethyl e do potencial de controle sobre algumas macrófitas aquáticas importantes, sugere-se que pesquisas com este produto continuem a ser feitas, com doses iguais ou maiores, por um período mais longo.

\section{LITERATURA CITADA}

ASOCIATION LATINOAMERICANA DE MALEZAS. Recomendaciones sobre unificación de los sistemas de evaluación em ensayos de control de malezas. ALAM, Bogotá, v. 1, p. 35-38, 1974.

BRANCO, S. M. Hidrobiologia aplicada à engenharia sanitária. 3.ed. São Paulo: CETESB, 1986. 616 p.

CARDOSO, L. R.; MARTINS, D.; TERRA, M. A. Sensibilidade a herbicidas de acessos de aguapé coletados em reservatórios do Estado de São Paulo. Planta Daninha, v. 21, p. 61-67, 2003. (Edição especial)

CARVALHO, F. T. et al. Plantas aquáticas e nível de infestação das espécies presentes no reservatório de Barra Bonita, no rio Tietê. Planta Daninha, Viçosa, v. 21, p. 15-19. 2003 (Edição especial)

GUIMARÃES, G. L. et al. Metodologia para avaliação de impacto ambiental de macrófitas em mesocosmos. Planta Daninha, v. 21, p. 37-42, 2003. (Edição especial)

HALLER, W. T. Options for mechanical and chemical aquatic weed control. In: WORKSHOP CONTROLE DE PLANTAS AQUÁTICAS, 1998, Brasília. Resumos... Brasília: Instituto Brasileiro de Meio Ambiente e dos Recursos Naturais Renováveis, 1998. p. 46-53.

MARTINS, D. et al. Controle químico de Pistia stratiotes, Eichhornia crassipes e Salvinia molesta em caixas d'água. Planta Daninha, v. 20, n. 1, p. 83-88, 2002.

PATTON, V. D.; STARNES, W. E. Aquatic weeds and water pollution. Hyacinth Control Journal, v. 8, n. 2, p. 48-49, 1970.

PITELLI, R. A. Macrófitas aquáticas no Brasil, na condição de problemáticas. In: WORKSHOP CONTROLE DE PLANTAS AQUÁTICAS, 1998, Brasília. Resumos... Brasília: Instituto Brasileiro de Meio Ambiente e dos Recursos Naturais Renováveis, 1998. p. 12-15.

SPRAYING SYSTEMS CO. Trop size versus accumulated volume percentage for XR TeeJet extended range $110^{\circ}$ series nozzles. Data Sheet, 12135-113. Wheaton, IL. 1992.

TANAKA, R. H. Eficácia de fluridone para o controle de Egeria spp. em caixas d'água e em represa de pequeno porte sem fluxo de água. 2001. $51 \mathrm{f}$. Dissertação (Mestrado em Agronomia/Agricultura) Universidade Estadual Paulista, Botucatu, 2001. 